\title{
Influence of Mullins effect on local strains and stresses in structures - Numerical analysis
}

\author{
L. Gornet \\ Ecole Centrale de Nantes - GeM. Nantes cedex, France
}

G. Chagnon

Laboratoire 3S. Université Joseph Fourier - INPG, Grenoble cedex, France

P. Charrier

Modvn Trellehorg. Zone ind. de Carquefou. Carquefou cedex, France

ABSTRACT: Elastomers exhibit a stress-softening response which depends on the maximum strain previously endured by the material. In complex structures, the strain is not homogeneous; as a consequence, the local maxima of strain is not uniform and the stress-softening either. In the sequel, a stress-softening model is used for finite element simulations. It appears that the damage distribution is not homogeneous and that weak zones of the structure vary in a great range. As expected, stress levels depend strongly on the loading path, and the strain state is also modified because of the non-homogeneity of the whole structure. This brings to light that the Mullins effect is of fundamental importance in structural and fatigue design.

\section{INTRODUCTION}

Elastomers exhibit a large number of phenomena as hysteresis, relaxation behaviour, stress-softening (Mullins, 1969). All these characteristics are of fundamental importance in the prediction of long-time behaviour. In this paper, we only focus on the stresssoftening phenomenon known as Mullins effect. Others phenomena are neglected. The rubber behaviour can be considered as time-independent and can be schematically represented by Figure 1, corresponding to a tensile cyclic loading. The virgin undamaged material is first stretched as the extension ratio reaches $\lambda_{I}$ and the stress follows path (I). Then the unloading from $\lambda_{I}$ to 0 follows path $\left(I^{\prime}\right)$. The second loading from 0 to $\lambda_{I I}>\lambda_{1}$ first follows path (I') until $\lambda=\lambda_{I}$ then it follows path (II). The second unloading from stretch ratio $\lambda_{\text {II }}$ to 0 follows path (II') which is different than the path $\left(\mathrm{I}^{\prime}\right)$. At a given stretch, the stress on $\left(\mathrm{II}^{\prime}\right)$ is lower than the stress on $\left(\mathrm{I}^{\prime}\right)$. Repeating this process, the loading path corresponding to the increase of stretch from 0 to $\lambda_{I I}$ is the path that joins (II') and the part (III) of the virgin curve. Finally, the corresponding unloading follows path (III').

During the life of the structure, numerous different cycles are imposed. A lot of work have been done for different materials to analyze the importance of the loading cycle sequences. The aim of this article is to

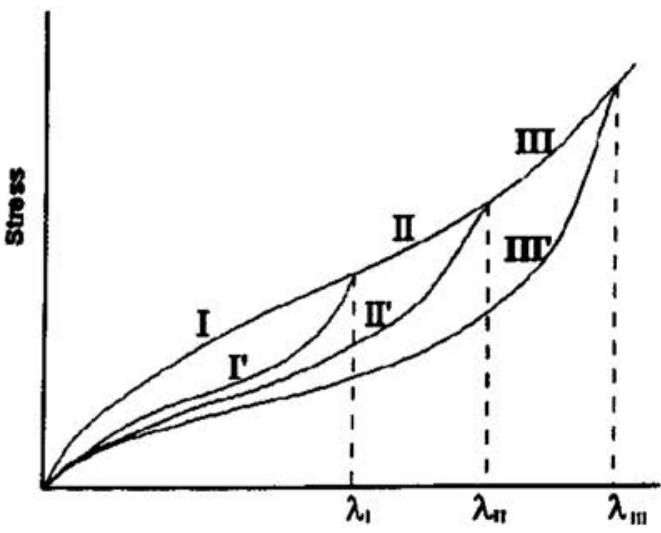

Figure 1. Schematic behaviour of a hyperelastic material with stress-softening.

show the influence of the primary loading cycles on the structure fatigue behaviour. As the local stress depends on the stress softening phenomenon, it is important to know what are the local stress and strain states after different loading histories.

In a first part, different models of stress-softening are presented. Then, the damage model used for predictions is recalled. In a second part, we show the influence 
of the loading history on an axisymmetrical notched sample dedicated to fatigue tests. In the last part, results on biaxial loading cases are evaluated. Finally, we conclude on the importance of the Mullins effect for fatigue studies.

\section{MODELS FOR THE MULLINS EFFECT}

Numerous models have been proposed since the stress softening phenomenon was pointed out. Models were based on material considerations. First, Mullins and Tobin (1957) considered that the material contains hard and a soft phases. Under loading the hard phase becomes soft phase. The evolution of the ratio of soft phase is studied and different evolution laws have been proposed, we can quote Johnson and Beatty (1993) or Zuñiga and Beatty (2002). Another approach is based on chain consideration, the interactions between chain and filler or chains with each other are studied, the first work was presented by Bueche $(1960,1961)$. Recently, Marckmann et al. (2002) have proposed a new constitutive equation to describe the evolution of chain length of the material. A last approach has an phenomenological point of view. By a study of the magnitude of the stress-softening according to the strain, Chagnon et al. (2004) have proposed a damage evolution law.

All these models have advantages and drawbacks, and a comparative study has been proposed by Chagnon et al. (2003). Here we choose to use the damage model of Chagnon et al. (2004) because of its ability to describe the Mullins effect, as well as its easy finite element implementation.

\subsection{Constitutive model}

The model used for predictions is based on the Continuum Damage Mechanics (Chagnon et al. (2004)). A thermodynamic variable $D$ is introduced to represent the stress-softening effect. This damage function is introduced within the hyperelastic energy density, it yields to:

$$
W=(1-D) W_{0}
$$

The damage evolution law is based on the ratio of stresses of the second loading curves. It is expressed in an exponential form:

$$
D=D_{\infty}\left(1-\exp \left(-\frac{I_{1}^{\max }}{\eta}\right)\right)
$$

thanks to the first strain invariant, where $D_{\alpha}$ and $\eta$ are material parameters. As it has been shown by Chagnon et al. (2004) parameters must be fitted on experimental data.
Table 1. Parameters of the damage model.

\begin{tabular}{lc}
\hline$C_{10}$ & $3,99 \mathrm{e}-2 \mathrm{MPa}$ \\
$C_{20}$ & $-4,05 \mathrm{E}-4 \mathrm{MPa}$ \\
$C_{30}$ & $1,31 \mathrm{E}-4$ \\
$D_{x}$ & 1,744 \\
$\eta$ & 3,85 \\
\hline
\end{tabular}

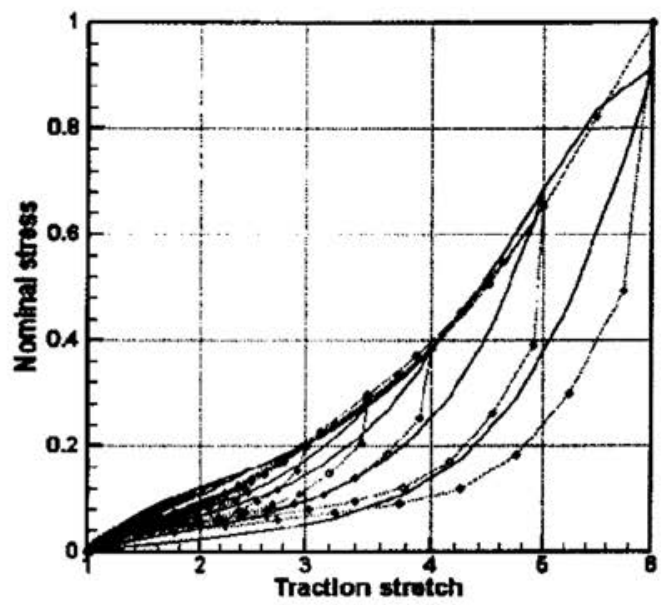

Figure 2. Simulation of a tensile test with the damage model of Chagnon et al. (2004) - (-) model, (..) experimental data.

The first loading curve is then described by both the hyperelastic behaviour and the damage function. It is important to choose a compatible strain energy function with regard to the damage evolution law. Energy densities with important hardening cause abnormal curvatures of the model; then, a regularized form of $W_{0}$ must be selected. The hyperelastic energy density proposed by Yeoh (1990) is chosen:

$$
W_{0}=C_{10}\left(I_{1}-3\right)+C_{20}\left(I_{1}-3\right)^{2}+C_{30}\left(I_{1}-3\right)^{3}
$$

The model is implemented in the finite element software Abaqus, thanks to the UMAT facility. The parameters are identified on experimental data corresponding to a carbon black natural rubber. The parameters values are given in Table 1.

The corresponding results are presented in Figures 4 and 5. The modelling is globally in good agreement with the experimental material behaviour.

\subsection{Finite elements implementation}

Here, we focus our attention on the implementation of the model in a finite element code. The numerical integration of the constitutive model is summarized in Table 2 . 
Table 2. Model implementation.

1. Given the deformation gradient $F_{n+1}$ Compute $B_{n+1}:=F_{n+1}\left(F_{n+1}\right)^{T}$

2. Compute the first strain invariant $I_{1}:=\operatorname{trace}\left(B_{n+1}\right)$

3. Check evolution damage

If $\mathrm{I}_{1}<\mathrm{I}_{1}{ }^{\max }$ then no damage evolution $\Rightarrow d_{n+1}:=d_{n}$ else

damage evolution $\Rightarrow d_{n+1}:=d\left(I_{1}\right)$ endif

4. Compute the Cauchy stress tensor $\sigma_{n+1}=-p I+2 B_{n+1} \frac{\partial W}{\partial B_{n+1}}$

5. Update damage criterion $\mathrm{I}_{1}{ }^{\text {max }}:=\max \left(\mathrm{I}_{1}, \mathrm{I}_{1}{ }^{\text {max }}\right)$

In order to make the finite element implementation at a low cost of computational times, it is important to evaluate the tangent operator of the model. The fourthorder tensor $\boldsymbol{C}$, known as the Lagrangian or material elasticity tensor is defined by the relation between Lagrangian strain $\mathbf{E}$ and Lagrangian stress $\mathbf{S}$ :

$\delta \mathbf{S}=\boldsymbol{C}: \delta \mathbf{E}$

It is defined by the spatial derivative as:

$$
\begin{aligned}
& C=\sum_{I, J, K, L=1}^{3} C_{J K L} \overrightarrow{E_{t}} \otimes \overrightarrow{E_{J}} \otimes \overrightarrow{E_{K}} \otimes \overrightarrow{E_{\iota}} \\
& C_{J K L}=\frac{\partial S_{\nu}}{\partial E_{K L}}=\frac{4 \partial^{2} W}{\partial C_{J} \partial C_{K L}}=C_{K L J}
\end{aligned}
$$

$\overrightarrow{\mathrm{E}_{\mathrm{I}}}$ are Lagrangian basis vectors. In order to take into account material incompressibility the relation (1) is modified in:

$$
\begin{aligned}
& W\left(I_{1}, I_{2}, J_{3}\right)=(1-D) W\left(I_{1}, I_{2}\right)+f(J) \\
& J=\operatorname{det}(F)
\end{aligned}
$$

The model can be dissociated into three parts for the calculus of tangent operator, the first one is hyperelastic behaviour, the second one, damage behaviour and the last one, incompressibility assumption.

\section{FATIGUE SPECIMEN PREDICTIONS}

The tests of crack propagation in fracture specimens conducted in this work are developed at ModynTrelleborg. Classically, fatigue tests are realized by a sequence of numerous identical cycles. The aim of the present part is to numerically applied what happens when a large cycle is realized somewhere in the succession of smaller cycles. The importance of the loading history for the structure is highlighted.

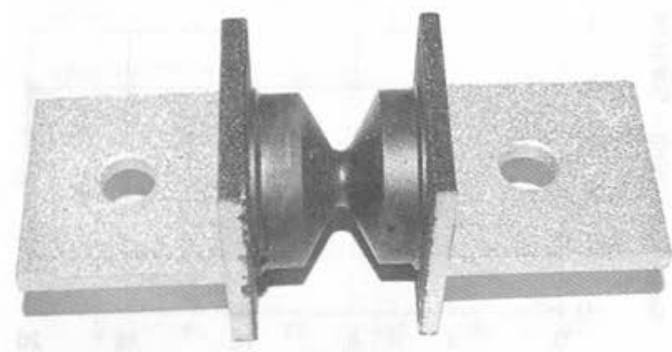

Figure 3. Axisymetrical notched specimen.

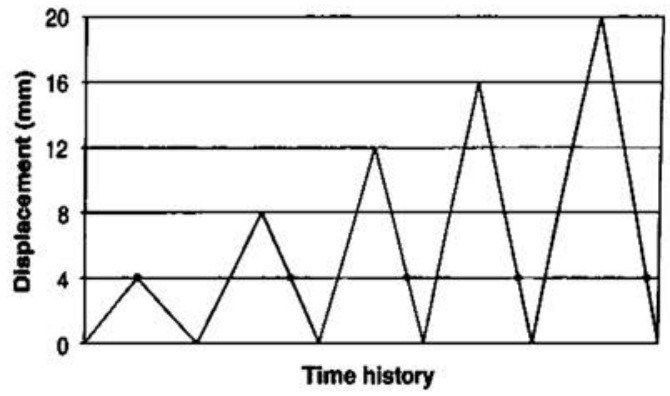

Figure 4. Traction cycle history imposed to the AE specimen.

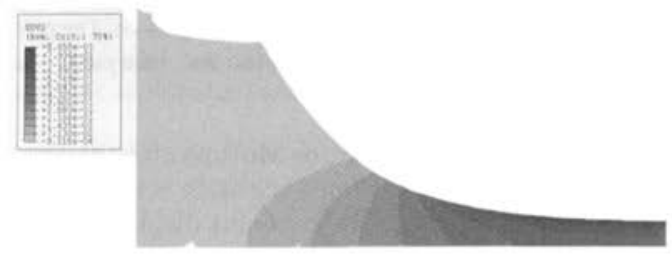

Figure 5. Damage distribution for the AE specimen after the $20 \mathrm{~mm}$ displacement cycle.

\subsection{Axisymetrical notched sample}

A first test is realized on the classical fatigue specimen called AE. It is represented in Figure 3.

\subsubsection{Tension test}

A uniaxial tensile simulation is carried out on Abaqus, with an axisymetric hypothesis. A sequence of increasing cycles is realized on the same specimen. It is depicted in Figure 4. The marks represent the compared structure states. Each point corresponds to the same imposed displacement with a different history, i.e. a different maximum imposed displacement to the structure.

As an example, the damage state after the largest displacement cycle is illustrated in Figure 5.

The central part of the specimen is the most stretched one. This region is the most favourable for 


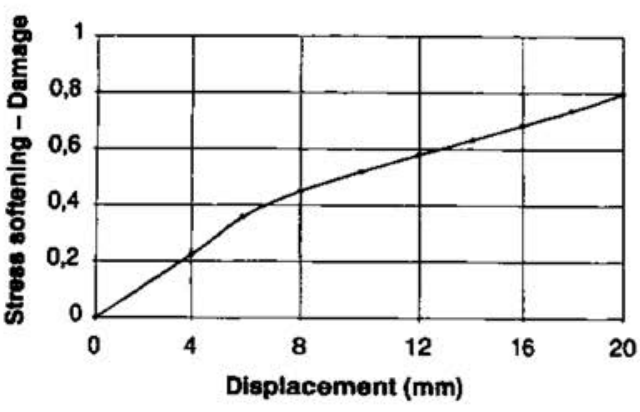

Figure 6. Evolution of the stress-softening according to the maximum imposed displacement.

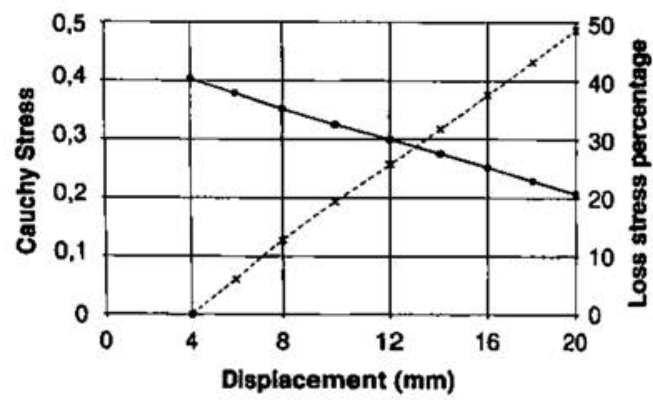

Figure 7. Evolution of the maximum principal stress in the centre of the structure after a larger cycle ( $\longrightarrow$ ). The percentage of stress loss is presented on the secondary axis -..).

crack initiation. The damage, or Mullins effect percentage, in the central zone of the specimen is represented according to the maximum imposed displacement in Figure 6. The evolution is quasi linear.

The central zone structural state stress remains uniaxial whatever the maximum imposed displacement, but the stress level is evolving. Figure 7 presents the evolution of the maximum principal stress in the structure for a $4 \mathrm{~mm}$ displacement after a maximum cycle of different size.

It appears that the stress loss rises $50 \%$ after a cycle of $16 \mathrm{~mm}$. It proves that the central structure endured two times less of stress. To finish the analysis, the strain state is compared. The structure geometry of the structure makes the central zone being in a quasi uniaxial tension. The evolution of the maximum principal elongation is presented in Figure 8 on the left hand side axis; the two other elongations are presented right hand side axis.

It appears that the structure is deformed more and more in the applied force direction. The rise is about $40 \%$. As a consequence, the strain in the two others directions is decreasing with the prescribed displacement.

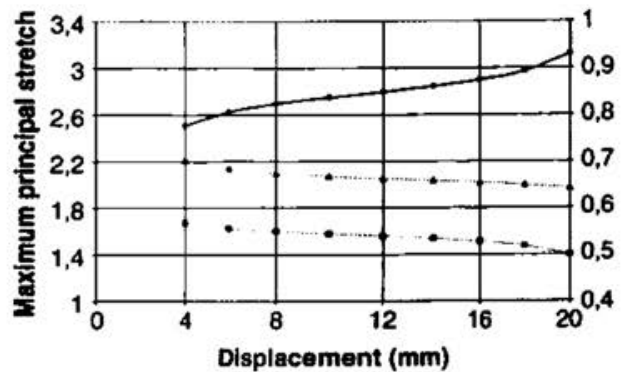

Figure 8. Evolution of the maximal principal elongation in the center of the structure after a larger cycle (-). Evolution of the two others principal elongations (-).

Table 3. Evaluation of the biaxiality ratio according to the maximum imposed displacement for the axisymetric cut specimen.

\begin{tabular}{llllll}
\hline Displacement & 4 & 6 & 8 & 10 & 12 \\
B & $-0,615$ & $-0,608$ & $-0,602$ & $-0,601$ & $-0,600$ \\
Displacement & 14 & 16 & 18 & 20 & \\
B & $-0,601$ & $-0,602$ & $-0,604$ & $-0,610$ \\
\hline
\end{tabular}

The deformation state of the body is fundamental for the fatigue analysis. The biaxiality state of deformation influences the crack apparition. Saintier (2000) has introduced a biaxiality coefficient $B$ to evaluate the deformation state of the material, its definition is:

$B=\frac{\ln \left(\lambda_{2}\right)}{\ln \left(\lambda_{1}\right)}$

Where $\lambda_{1}$ and $\lambda_{2}$ are extensions on the considered surface. The evaluation of this ratio for the previous example is given by Table 3 .

It is interesting to note that the biaxiality ratio does not evolve whatever is the maximum prescribed displacement. It remains close to -0.5 which corresponds to an uniaxial tension state.

The stress-soflening imposed to the structure implies a fall of the stress in the structure (about $40 \%$ ) and a large modification of extension amplitudes. But the strain state does not evolve for a constant biaxiality ratio.

\subsubsection{Torsion test}

In the same way as for the traction tests, a parametric analysis is performed for important cycles on torsion behaviour of an AE specimen. The behaviour of the specimen is studied under torsion angle of $30^{\circ}$ but after different maximum cycles between $30^{\circ}$ and $225^{\circ}$. The damage concentrates in the central zone of the structure, as shown in Figure 9 after a loading torsion cycle of $225^{\circ}$. 


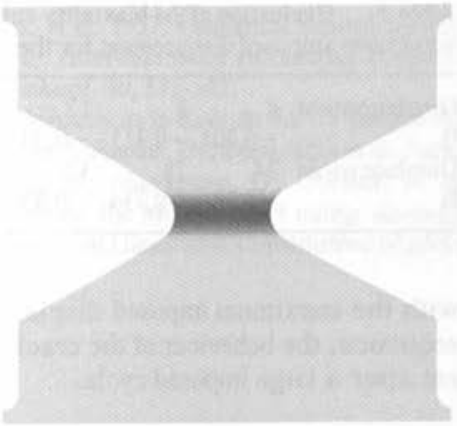

Figure 9. Damage state of the axisymetric notched specimen (AE) after a $225^{\circ}$ rotation cycle.

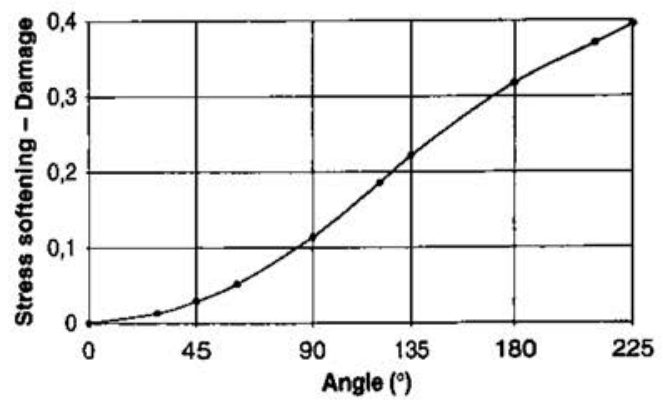

Figure 10. Evolution of damage in the center of the structure according to the maximum prescribed angle.

The maximum damage in the central zone of the structure is plotted according to the maximum angle imposed to the specimen in Figure 10. The damage is maximum at the surface of the specimen. It appears that the stress-softening varies quasi-linearly with the torsion angle imposed to the structure.

As a consequence, the stress state is modified by the damage evolution. The evolution of the maximum principal stress in the centre of the structure is presented in Figure 11. The stress loss rises $20 \%$ for the largest imposed angle.

The evolution of the principal stretches is presented in Figure 12.

As for uniaxial tests prescribed, the elongation in the most sollicitated direction increases whereas the two others decrease. It is interesting to evaluate the evolution of the biaxiality ratio; it is presented in Table 4. It appears that $B$ does not depend on the maximum imposed angle. It remains equal to -0.92 which is near a pure shear strain field.

\subsection{Centre cracked plate}

A second test is realized on a classical fatigue structure: a cracked plate ( $40 \mathrm{~mm}$ large and $60 \mathrm{~mm}$ high)

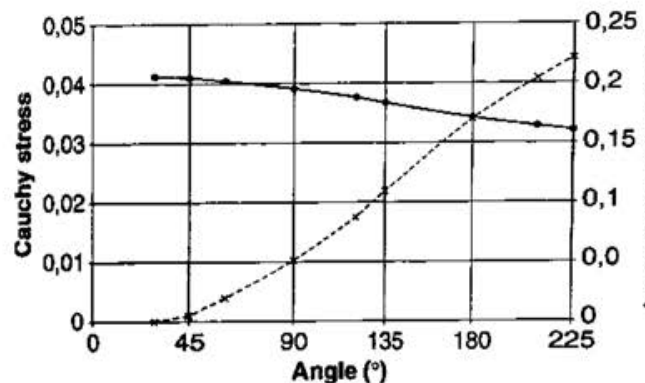

Figure 11. Evolution of the maximal principal stress in the center of the structure according to the maximum imposed angle.

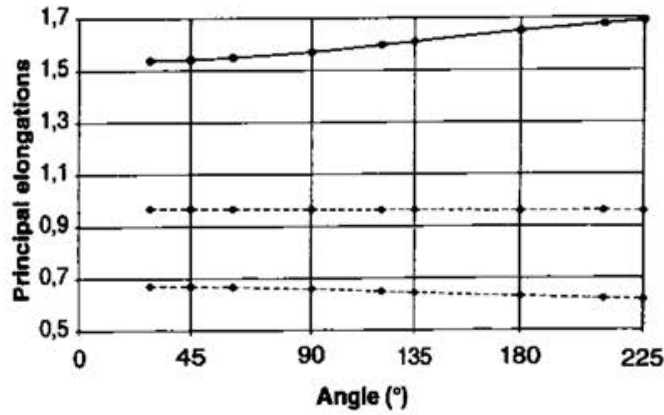

Figure 12. Evolution of the maximal principal elongation (-) and the two others principal elongations (--) in the center of the structure after a larger cycle.

Table 4. Evaluation of the biaxiality ratio according to the maximum imposed angle for the axisymetric cut specimen.

\begin{tabular}{llllll}
\hline Angle & 30 & 45 & 60 & 90 & 120 \\
B & $-0,926$ & $-0,926$ & $-0,925$ & $-0,922$ & $-0,921$ \\
Angle & 135 & 180 & 210 & 225 & \\
B & $-0,922$ & $-0,920$ & $-0,918$ & $-0,918$ & \\
\hline
\end{tabular}

with a horizontal centred crack of $2.5 \mathrm{~mm}$ long. The structure is studied for a $4 \mathrm{~mm}$ displacement, after different maximum cycles at displacement between 4 and $40 \mathrm{~mm}$. The damage distribution of the structure after a $40 \mathrm{~mm}$ imposed displacement is presented in the Figure 13. It appears that the damage (i.e. the stresssoftening phenomenon) is concentrated at crack tip. It is about three times the stress softening of the rest of the structure.

The structure becomes totally non-homogeneous, the behaviour of the crack tip is very different than the one in the rest of the plate. Moreover, local data of the crack tip are largely modified. The evolution of the maximum principal stress is shown in Figure 14. 


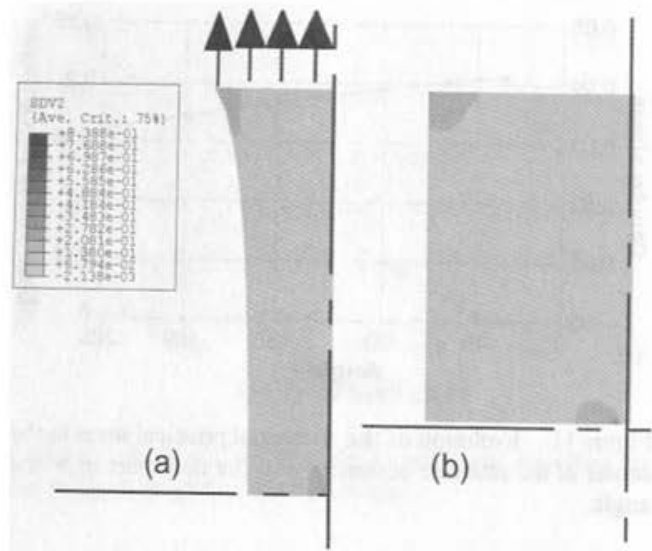

Figure 13. Stress softening map of the crack plate after a $40 \mathrm{~mm}$ cycle displacement - (a) deformed state - (b) nondeformed state.

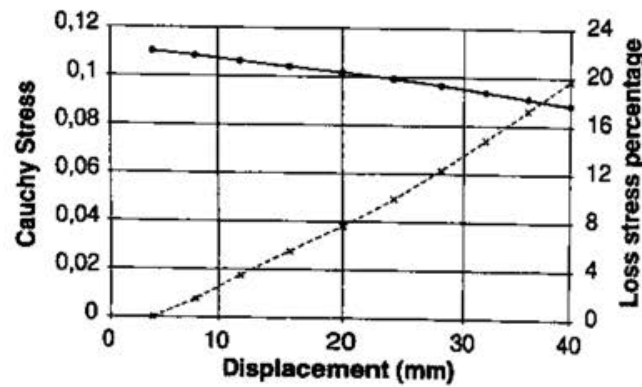

Figure 14. Evolution of the maximum stress at the crack tip.

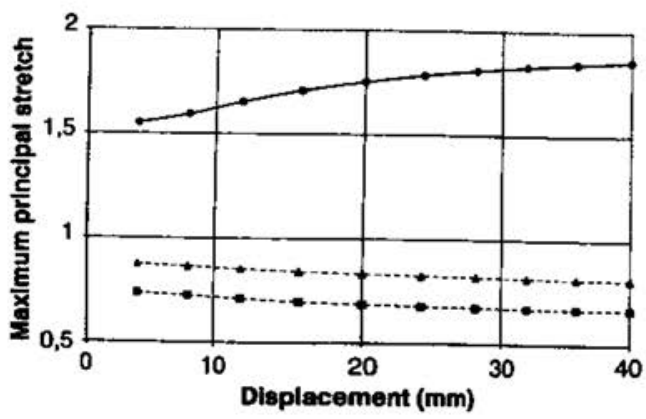

Figure 15. Evolution of the maximal principal elongation (--) and the two others principal elongations (--) at the crack tip after a larger imposed cycle.

As for the AE specimen, the strain field is modified. Figure 15 illustrates the variations of the principal stretches with the maximum prescribed displacement.

Nevertheless, variations of the biaxiality ratio are presented in the Table 5. It appears that the ratio evolves
Table 5. Evaluation of the biaxiality ratio according to the maximum imposed displacement for the centred crack plate.

\begin{tabular}{llllll}
\hline Displacement & 4 & 8 & 12 & 16 & 20 \\
B & $-0,305$ & $-0,311$ & $-0,317$ & $-0,323$ & $-0,327$ \\
Displacement & 24 & 28 & 32 & 36 & 40 \\
B & $-0,331$ & $-0,334$ & $-0,337$ & $-0,339$ & $-0,342$ \\
\hline
\end{tabular}

with the maximum imposed displacement. As a consequence, the behaviour of the crack tip can be different after a large imposed cycle.

\section{CONCLUSIONS}

The numerical analysis of three examples (two on a fatigue test specimen and one on a cracked structure) demonstrates that the structure becomes non-homogeneous after stressing. The analysis of the critical fields highlights that the stress decreases, whereas the strain becomes larger. In fact, the decrease of the stress field leads to a concentration of the deformation in the weaker zone of the structure. The measure of the biaxiality ratio is an appropriate tool to analyse the strain field of a given free surface. The two first examples show that it does not evolve; but in the last example, some variations are observed. It proves that according to the geometry of the considered structure, the Mullins effect induces different behaviours of the structural critical zone.

\section{REFERENCES}

Bueche, F. 1960. Molecular basis for the Mullins effect. J. Appl. Polym. Sci. 3, 107-114.

Bueche, F. 1961. Mullins effect and rubber filler interaction. J. Appl. Polym. Sci. 5, 271-281.

Chagnon, G., Verron, E., Gornet, L., Marckmann, G. \& Charrier, P. 2004. On the relevance of continuum damage mechanics as applied to the Mullins. J. Mech. Phys. Solids 52, 1627-1650.

Chagnon, G., Verron, E., Marckmann, G., Gornet, L. \& Charrier, P. 2003. Comparison of recent models for the Mullins effect: formulation and finite element implementation, Constitutive Models for Rubber III, J.J.C. Busfield and A.H. Muhr eds, 245-250.

Johnson, M.A. \& Beatty, M.F. 1993. The Mullins effect in uniaxial extension and its influence on transverse vibration of rubber string. Continuum Mech. Thermodyn.5, 83-115.

Marckmann, G., Verron, E., Gornet, L., Chagnon, G., Charrier, P. \& Fort, P. 2002. A theory of network alteration for the Mullins effect. J. Mech. Phys. Solids 50, 2011-2028.

Miehe. C. 1995. Discontinuous and continuous damage evolution in Ogden type large strain elastic materials. Eur. $J$. Mech., A/Solids 14, 697-720.

Mullins, L. 1969. Softening of rubber by deformation Rubber Chem. Technol. 42, 339-362. 
Mullins, L. \& Tobin, N.R. 1957. Theoretical model for the elastic behavior of filler-reinforced vulcanized rubbers. Rubber Chem. Technol. 30, 551-571.

Saintier, N. 2000. Prévisions de la durée de vie en fatigue du NR sous chargement multiaxial. PhD Thesis Mines de Paris.

Verron, E., Chagnon. G., Marckmann, G., Gornet, L. \& Charrier, P. Modelling the Mullins effect using damage mechanics: efficiency and limitations. Constitutive Models for Rubber III, J.J.C. Busfield and A.H. Muhr eds, 237-243.

Yeoh, O.H. 1990. Characterization of elastic properties of carbon black filled rubber vulcanizates. Rubber Chem. Technol. 63, 792-805.

Zuniga, A.E. \& Beatty, M.F. 2002. A new phenomenological model for stress-softening in elastomers. Z. angew. Math. Phys. 53, 794-814. 